\title{
A Framework for the Stabilization of General Nonholonomic Systems With an Application to the Plate-Ball Mechanism
}

\author{
Giuseppe Oriolo, Senior Member, IEEE, and Marilena Vendittelli
}

\begin{abstract}
We present a framework for the stabilization of nonholonomic systems that do not possess special properties such as flatness or exact nilpotentizability. Our approach makes use of two tools: an iterative control scheme and a nilpotent approximation of the system dynamics. The latter is used to compute an approximate steering control which, repeatedly applied to the system, guarantees asymptotic stability with exponential convergence to any desired set point, under appropriate conditions. For illustration, we apply the proposed strategy to design a stabilizing controller for the plate-ball manipulation system, a canonical example of nonflat nonholonomic mechanism. The theoretical performance and robustness of the controller are confirmed by simulations, both in the nominal case and in the presence of a perturbation on the ball radius.
\end{abstract}

Index Terms-Iterative steering (IS), nilpotent approximations (NAs), nonholonomic systems, plate-ball mechanism, stabilization.

\section{INTRODUCTION}

$\mathbf{T}$ HE PROBLEMS of planning and controlling motions of nonholonomic systems have been the subject of very intensive research over the last two decades. This burst of activity, first motivated by the theoretical challenges posed by these systems (the most notable being the impossibility of achieving stabilization by smooth feedback [4]), was supported by the practical relevance of nonholonomic behavior, which arises, and must be dealt with, in many advanced robotic systems, such as wheeled mobile robots, dextrous manipulation mechanisms, and space robots.

In many cases, nonholonomic robots are adequately represented by their kinematic model (i.e., assuming pseudovelocities as control inputs), so that a driftless dynamic system can be considered for control synthesis. For example, this is the case of wheeled mobile robots. In the following literature review, as well as in all our developments, we shall, therefore, refer to nonholonomic systems without drift.

For nonholonomic systems that can be made nilpotent by a feedback transformation, the problem of generating a feasible path (and the associated steering control) connecting two arbitrary configurations has been solved by differential geometric

Manuscript received May 19, 2004. This paper was recommended for publication by Associate Editor K. Lynch and Editor H. Arai upon evaluation of the reviewers' comments. This paper was presented in part at the International Conference on Robotics and Automation, Seoul, Korea, May 2001.

The authors are with the Dipartimento di Informatica e Sistemistica, Università di Roma “La Sapienza,”00184 Rome, Italy (e-mail: oriolo@dis.uniroma1. it; vendittelli@dis.uniroma1.it).

Digital Object Identifier 10.1109/TRO.2004.839231 arguments in [15]. Other cases of special interest are chainedform transformable [32] systems (a particular case of nilpotentizability) and flat [9] systems; for these two classes, which coincide in the two-input case, many efficient steering techniques exist (see [8] for a review).

The problem of stabilizing a given configuration has been solved for chained forms using time-varying and/or discontinuous feedback laws, respectively pioneered in [38] and [41]. While many other techniques have been subsequently proposed to improve the performance of these basic approaches (see [24] for an excellent review), systematic and effective design procedures are only available for special classes of driftless dynamics. In addition to flat and chained-form transformable systems, these include low-dimensional systems [14], and the case in which the Lie algebra has a particular growth structure [36].

However, there exist nonholonomic robots, referred to as general in this paper, whose kinematic model does not fall into any of the aforementioned classes. For example, mobile robots with more than one trailer cannot be transformed in chained form, unless each trailer is hinged to the midpoint of the previous wheel axle-a particular arrangement, very unusual in real trailer vehicles, known as "on-hooking." Another such example are robotic systems that perform object manipulation by rolling contacts [31]: even the simplest mechanism in this category, the so-called plate-ball system, does not admit a chained-form transformation. More in general, for two-input systems, as soon as the dimension of the state space reaches five, exact nilpotentizability becomes the exception rather than the rule (whereas all systems up to dimension four possess this property [29]).

With reference to path planning, techniques for steering nonnilpotentizable systems include an iterative version of the method in [15], the continuation method of [43], and the generic loop method of [40]. The problem of generating an open-loop control steering a driftless system along a generic path has been solved in [17] through a sequence of highly oscillatory controls that converge to the desired input.

Coming to the stabilization problem for general driftless dynamics, Coron [6] has shown that controllability implies stabilizability by continuous time-varying feedback, a path-breaking result which, however, does not explicitly provide a constructive design procedure; a preliminary result of the same flavor was given in [13]. In [24], a technique is presented that allows transforming a given smooth stabilizing law into a nonsmooth 
controller which achieves exponential convergence. Stabilizing controllers making use of Liu's steering inputs [17] were presented in [26] and [27]; the latter approach, which is applicable to sufficiently regular driftless systems, relies on an iteration mechanism for achieving robustness to unmodeled dynamics. Another technique based on iteration is averaging. In [45], it is shown how to obtain exponential stability of the averaged system for driftless dynamics.

The objective of this paper is to present a new framework for the stabilization of general nonholonomic systems. Our basic idea is to apply the iterative steering (IS) paradigm of [19], which shows that asymptotic stabilization of nonlinear systems can be achieved by the repeated feedback application of approximate steering controls that possess certain properties. To design such controls, we rely on nilpotent approximations (NAs) [10], [2], high-order local approximations of control systems that are useful when tangent linearization does not retain controllability, as in nonholonomic systems. NAs have already been implicitly used in some of the aforementioned papers, e.g., see [15] and [17]. Here, we use them explicitly, in the sense that our steering controllers are, in fact, designed and computed on the NA of the original system. This is made easy by the fact that NAs are polynomial and triangular. It is then possible to integrate their equations in closed form under parameterized inputs.

The combination of the above two tools results in a strategy which is applicable to any driftless controllable system. In particular, a general control algorithm is obtained, which guarantees asymptotic stability with exponential convergence rate. In addition, the adoption of the iterative steering approach provides desirable robustness properties with respect to unmodeled dynamics, i.e., rejection of small nonpersistent perturbations and ultimate boundedness of the error in the presence of persistent perturbations.

As an illustrative case study, we consider the plate-ball manipulation system. Following the proposed design procedure, an explicit control algorithm is constructed that asymptotically stabilizes the ball at any desired configuration, with the expected exponential convergence. Simulation results are presented both in nominal conditions, to confirm the theoretical performance, and in perturbed conditions (i.e., when the radius of the ball is not known) to highlight the robustness gained by our feedback scheme.

The paper is organized as follows. After stating the considered problem in Section II, we offer in Section III a short review of our basic tools, i.e., iterative steering and nilpotent approximations. Such a review provides the essential context for the developments in the paper, but at the same time, directs the reader to the relevant literature in the area. Our stabilization strategy is described in Section IV. In particular, we present an algorithm for designing appropriate steering laws via NA, the control algorithm based on the iteration of such steering laws, and the associated stability proof; at this point, we also provide a comparative discussion of our method with respect to related techniques. In Section V, the proposed stabilization framework is applied to the plate-ball system, providing an explicit control algorithm whose effectiveness is theoretically proven and confirmed by simulations. Further areas of research are mentioned in the concluding section.

\section{PROBLEM FORMULATION}

We consider general driftless nonholonomic systems in the form

$$
\dot{x}=\sum_{i=1}^{m} g_{i}(x) u_{i}
$$

where $g_{1}, \ldots, g_{m}$ are smooth vector fields on $\mathbb{R}^{n}, x \in \mathbb{R}^{n}$ is the state vector, and $u=\left(u_{1}, \ldots, u_{m}\right) \in \mathbb{R}^{m}$ is the control vector. General means that no special property is assumed, other than controllability, i.e., the fact that the Lie algebra associated to the vector fields $g_{i}$ is of rank $n$ (Lie algebra rank condition). In particular, we shall not require system (1) to be flat or exactly nilpotentizable.

The class of general nonholonomic systems includes some interesting robotic systems, such as multifingered hands for manipulation and off-hooked trailer systems. It is exactly at these challenging mechanisms that our investigation is aimed. ${ }^{1}$ Our objective is to build a controller that asymptotically stabilizes the origin of the state space, assumed without loss of generality to be the desired equilibrium.

\section{BASIC TOOLS}

The objective of this section is to present some background material on the two basic tools of our stabilization method, i.e., the IS technique and the NA procedure.

\section{A. Iterative Steering}

The IS technique can be used to achieve stabilization of nonlinear systems (with or without drift) through the repeated application of a suitable steering control. In view of our interest in kinematic nonholonomic mechanisms, we summarize below the basic features of this technique, as applied to the driftless system (1), referring the reader to [19] for details.

Consider a generic initial condition $x_{0} \in B$, an open ball around the origin of the state space of system (1). Assume that a finite-time steering control $u(t)=\alpha\left(x(t), x_{0}, t\right), t \in[0, T]$ satisfying the following condition is available.

Condition 1: The steering control $\alpha$ is such that:

a) $\alpha(x, 0, t)=0$ for any $(x, t) \in \mathbb{R}^{n} \times[0, T]$;

b) $\alpha$ is locally Lipschitzian in $x$, piecewise-continuous in $t$, for $t \in[0, T]$, and Hölder-continuous of order $p$ in $x_{0}$ at the origin, i.e.,

$$
\left|\alpha\left(0, x_{0}, t\right)\right| \leq \mu\left|x_{0}\right|^{p}, \quad t \in[0, T], \quad \mu>0, \quad p>0
$$

c) contraction is guaranteed

$$
|x(T)| \leq \beta\left|x_{0}\right|, \quad \beta<1, \quad \forall x_{0} \in B .
$$

Here, the symbol $|\cdot|$ denotes the Euclidean norm.

In principle, one may specify $\alpha$ either in feedforward (i.e., as a function of $x_{0}$ and $t$ only) or in feedback. In the first case, the steering control $\alpha$ may be the output of a path planner. The second includes the situation in which a preliminary feedback transformation is used to put the system in a canonical form

\footnotetext{
${ }^{1}$ The stabilization strategy used in this paper clearly works for nilpotentizable systems as well, with the further simplification coming from the fact that the use of NAs is obviously no longer necessary; see [19] for an application to chained forms.
} 
convenient for planning (e.g., a chained form), but also in the case of a true, error-based feedback steering law.

Consider now the sequence of time instants $\left\{t_{k}=k T\right\}$, with $k=0,1,2, \ldots$, and the associated time intervals $I_{k+1}=$ $\left[t_{k}, t_{k+1}\right]$, hereafter called iterations. In each iteration, define the control input as

$$
u(t)=\alpha\left(x(t), x_{k}, t-t_{k}\right), \quad t \in I_{k+1}
$$

i.e., update the above steering control $\alpha$ on the basis of $x_{k}=$ $x\left(t_{k}\right)$, the initial state of the iteration. For $t \in[0, \infty)$, (4) defines a feedback law which depends on the measured state, at least at sampled time instants.

The result below, proven in [19], characterizes the stability properties of the origin of system (1) under IS.

Theorem 1: If the steering control $\alpha$ satisfies Condition 1, its iterative application (4) renders the origin of system (1) asymptotically stable, with an exponential convergence rate that is proportional to $|\log \beta|$. The asymptotic stability is global, provided that $B=\mathbb{R}^{n}$ in (3).

Note the following points.

- In the above result, asymptotic stability is to be intended in the sense of Lyapunov: $x(t)$ can be arbitrarily bounded for all $t \in[0, \infty)$ by bounding $x_{0}$, and converges to the origin as $t \rightarrow \infty$.

- If Condition 1 is modified by requiring simple continuity (in place of Hölder-continuity) of $\alpha$ in $x_{0}$, uniform asymptotic stability is preserved, but the convergence rate may not be exponential.

- If $p \geq 1$, then the origin is exponentially stable in the standard sense. Otherwise, it is $\rho$-exponentially stable, according to the definition of [14].

Another benefit of the IS stabilization technique is that it provides a degree of robustness against additive perturbations. Small nonpersistent perturbations are rejected, while ultimate boundedness of the error is guaranteed under persistent perturbations. In this paper, we shall not address the robustness issue from a theoretical viewpoint; we refer the reader to [19, Th. 2] for a precise characterization of the relevant conditions.

To apply the IS paradigm to a specific control system, one must be able to compute a steering control $\alpha\left(x, x_{0}, t\right)$ that satisfies Condition 1. As we are dealing with general nonholonomic systems, powerful properties such as flatness or nilpotency cannot be exploited. On the other hand, an exact planner is not required. Equation (3) indicates that we may settle for an approximate planner, as long as its performance is provably good. These two circumstances call for a new approach, and this is exactly where NAs come into play.

\section{B. Nilpotent Approximations}

A nilpotent approximation of the control system (1) may be thought of as a generalization of the linear approximation. With respect to the latter, NAs are higher order approximations with an increased degree of adherence to the original dynamics, in the sense that they preserve the structural properties (including controllability) of the original system. Various researchers have developed techniques for computing NAs (e.g., [2] and [10]). In the following, we recall some basic material following closely the approach in [2].
Fix a point $\bar{x} \in \mathbb{R}^{n}$ and let $L^{s}(\bar{x})$ be the vector space generated by the values at $\bar{x}$ of the Lie brackets of $g_{1}, \ldots, g_{m}$ of length $\leq s, s=1,2, \ldots$ (input vector fields are brackets of length one). Controllability of system (1) guarantees that there exists a smallest integer $r=r(\bar{x})$ such that $\operatorname{dim} L^{r}(\bar{x})=n$. This integer is called the degree of nonholonomy at $\bar{x}$. Let $n_{s}(\bar{x})=$ $\operatorname{dim} L^{s}(\bar{x}), s=1, \ldots, r$, and define the growth vector at $\bar{x}$ as $\left(n_{1}(\bar{x}), \ldots, n_{r}(\bar{x})\right)$.

In association with system (1), consider now a smooth realvalued function $f$. Call first-order nonholonomic partial derivatives of $f$ the Lie derivatives $g_{i} f$ of $f$ along $g_{i}, i=1, \ldots, m$. Call $g_{i}\left(g_{j} f\right), i, j=1, \ldots, m$, the second-order nonholonomic partial derivatives of $f$, and so on.

Definition 1: A function $f$ is said to be of order $\geq s$ at a point $\bar{x}$ if all its nonholonomic partial derivatives having order $\leq s-1$ vanish at $\bar{x}$. If $f$ is of order $\geq s$ and not of order $\geq s+1$ at $\bar{x}$, it is said to be of order $s$ at $\bar{x}$.

Definition 2: A vector field $h$ is said to be of order $\geq q$ at $\bar{x}$ if, for every $s$ and every function $f$ of order $s$ at $\bar{x}$, function $h f$ has order $\geq q+s$ at $\bar{x}$. If $h$ is of order $\geq q$ but not $\geq q+1$, it is said to be of order $q$ at $\bar{x}$.

From the definitions above, it follows that input vector fields $g_{i}, i=1, \ldots, m$, have order $\geq-1$.

Definition 3: A system

$$
\dot{\hat{x}}=\sum_{i=1}^{m} \hat{g}_{i}(\hat{x}) u_{i}
$$

is a nilpotent approximation ${ }^{2}$ of system (1) at $\bar{x}$ if:

- the vector fields $g_{i}-\hat{g}_{i}$ are of order $\geq 0$ at $\bar{x}$;

- its Lie algebra is nilpotent of step $s>r(\bar{x})$, i.e., all Lie brackets of length greater than $s$ vanish.

Note that the first property implies the preservation of growth vector and controllability.

Algorithms for computing NAs are based on the existence at each point of a set of locally defined privileged coordinates.

Definition 4: Let the integer $w_{j}, j=1, \ldots, n$ be defined by setting $w_{j}=s$ if $n_{s-1}<j \leq n_{s}$, with $n_{s}=n_{s}(\bar{x})$ and $n_{0}=0$. Local coordinates $z_{1}, \ldots, z_{n}$ centered at $\bar{x}$ form a system of privileged coordinates if the order of $z_{j}$ at $\bar{x}$ equals $w_{j}$, for $j=$ $1, \ldots, n$. In this case, $w_{j}$ is called the weight of coordinate $z_{j}$.

A procedure for constructing a set of privileged coordinates is given in [2]; for an explicit formula, see [47]. While we do not give details of such a procedure, there are some structural properties of privileged coordinates that we shall need in the paper.

Denote by $A$ the inverse of a matrix whose columns are a basis at $\bar{x}$ of $L^{r}(\bar{x})=\mathbb{R}^{n}$, and let $y=A(x-\bar{x})$. Privileged coordinates at $\bar{x}$ are always obtained by a polynomial change of coordinates of the form

$$
\begin{aligned}
z_{1} & =y_{1} \\
z_{2} & =y_{2}+\operatorname{pol}_{2}\left(y_{1}\right) \\
z_{3} & =y_{3}+\operatorname{pol}_{3}\left(y_{1}, y_{2}\right) \\
& \vdots \\
z_{n} & =y_{n}+\operatorname{pol}_{n}\left(y_{1}, \ldots, y_{n-1}\right)
\end{aligned}
$$

${ }^{2}$ This definition is equivalent to that given in [2] and [11]. 
where $\operatorname{pol}_{j}(\cdot), j=2, \ldots, n$ denotes a polynomial function of $y_{1}, \ldots, y_{j-1}$ that includes only terms of degree $\geq 2$ and $<w_{j}$. In compact form, we may write

$$
z=\phi_{\bar{x}}(x)=y+\operatorname{pol}(y)=A(x-\bar{x})+\operatorname{pol}(A(x-\bar{x})) .
$$

From the triangular structure of (5), it is immediate to see that the inverse change of coordinates from $z$ to $y$ has exactly the same form of (6)

$$
y=z+\operatorname{pol}^{\prime}(z) .
$$

In particular, $\operatorname{pol}^{\prime}(\cdot)$ and $\operatorname{pol}(\cdot)$ only differ for the value of the coefficients. Hence, the inverse mapping from $z$ to $x$ is obtained as

$$
x=\phi_{\bar{x}}^{-1}(z)=\bar{x}+A^{-1}\left(z+\operatorname{pol}^{\prime}(z)\right) .
$$

As before, the $j$ th component $\operatorname{pol}_{j}^{\prime}(\cdot)$ of $\operatorname{pol}^{\prime}(\cdot)$ is a polynomial function of $z_{1}, \ldots, z_{j-1}$ that includes only terms of degree $\geq 2$ and $<w_{j}$.

The order of functions and vector fields expressed in privileged coordinates can be computed in an algebraic way.

- The order of the monomial $z_{1}^{\gamma_{1}} \ldots z_{n}^{\gamma_{n}}$ is equal to its weighted degree $w(\gamma)=w_{1} \gamma_{1}+\cdots+w_{n} \gamma_{n}$.

- The order of a function $f(z)$ at $z=0$ (the image of $\bar{x}$ ) is the least-weighted degree of the monomials actually appearing in the Taylor expansion of $f$ at zero.

- The order of a vector field $h(z)=\sum_{j=1}^{n} h_{j}(z) \partial_{z_{j}}$ at $z=$ 0 is the least-weighted degree of the monomials actually appearing in the Taylor expansion of $h$ at zero

$$
h(z) \sim \sum_{\gamma, j} a_{\gamma, j} z_{1}^{\gamma_{1}} \ldots z_{n}^{\gamma_{n}} \partial_{z_{j}}
$$

considering the term $a_{\gamma, j} z_{1}^{\gamma_{1}} \ldots z_{n}^{\gamma_{n}} \partial_{z_{j}}$ as a monomial and assigning to $\partial_{z_{j}}$ the weight $-w_{j}$.

Once a set of privileged coordinates $z$ has been built, an NA can be obtained as follows.

1) Express the dynamics of the original system in privileged coordinates

$$
\dot{z}=\sum_{i=1}^{m} g_{i}(z) u_{i} .
$$

2) Expand vector fields $g_{i}(z)$ in Taylor series at zero, express them in terms of vector fields homogeneous with respect to the weighted degree

$$
g_{i}(z)=g_{i}^{(-1)}(z)+g_{i}^{(0)}(z)+g_{i}^{(1)}(z)+\cdots
$$

and let $\hat{g}_{i}(z)=g_{i}^{(-1)}(z)$. By construction, the $j$ th component $\hat{g}_{i j}$ of $\hat{g}_{i}$ depends only on $z_{1}, \ldots, z_{j-1}$.

3) Define the approximate system as

$$
\dot{\hat{z}}_{j}=\sum_{i=1}^{m} \hat{g}_{i j}\left(\hat{z}_{1}, \ldots, \hat{z}_{j-1}\right) u_{i}, \quad j=1, \ldots, n
$$

where the $\hat{g}_{i j}$ 's are the components of $\hat{g}_{i}$.

System (9) is an NA of the original dynamics (1). We emphasize its properties that are essential for our purposes.
- The $\hat{g}_{i j}$ 's are homogeneous polynomials of weighted degree $w_{j}-1$.

- The remainder terms $r_{i j}(z)=g_{i j}(z)-\hat{g}_{i j}\left(z_{1}, \ldots, z_{j-1}\right)$ $(i=1, \ldots, m, j=1, \ldots, n)$ of the Taylor series expansion of $g_{i j}(z)$ are functions of order $w_{j}$ at zero.

- System (9) is strictly triangular.

\section{The Stabilization StRategy}

As already mentioned, our basic idea is to achieve asymptotic stabilization of general nonholonomic systems through the IS paradigm, using NAs to derive steering controls that satisfy Condition 1. We formalize this idea in this section, first clarifying the steps of the design procedure, and then providing a control algorithm whose correctness is proved.

\section{A. Design of a Steering Controller}

To obtain a stabilization strategy based on the IS approach, we need an appropriate steering controller. To this end, we propose the following Design Algorithm.

Step D1) Build an NA of system (1) at a generic point $\bar{x}$ following the procedure briefly recalled in Section III-B. One obtains a nilpotent system $\Psi_{\bar{x}}$ of the form (9), where the $z$ are privileged coordinates centered at $\bar{x}$ (i.e., the image of $\bar{x}$ is zero), and obtained from $x$ through a polynomial change of coordinates $z=\phi_{\bar{x}}(x)$.

Step D2) For a generic $z^{f} \in \mathbb{R}^{n}$ (a target point for $\Psi_{\bar{x}}$ ), devise an open-loop steering controller $\alpha\left(z^{f}, t\right), t \in$ $[0, T]$ that satisfies the following condition.

Condition 2: The steering control $\alpha$ is such that:

a) $\alpha(0, t)=0$, for any $t \in[0, T]$;

b) $\alpha$ is piecewise-continuous in $t$, for $t \in[0, T]$, and Hölder-continuous of order $p$ in $z^{f}$ at the origin, i.e.,

$$
\left|\alpha\left(z^{f}, t\right)\right| \leq \mu\left|z^{f}\right|^{p} \quad t \in[0, T] \quad \mu>0 \quad p>0
$$

c) the state of $\Psi_{\bar{x}}$ is steered from zero (the image of $\bar{x}$ ) to $z^{f}$ in time $T$, i.e., $z(T)=z^{f}$.

To perform Step D2, one may use, in general, the systematic method for steering nilpotent systems proposed in [15]. The basic idea of this technique is to build an extended system by augmenting the original dynamics with an appropriate set of Lie brackets of the input vector fields, in such a way that computing a steering control for this system is straightforward. The solution is then mapped back to the original system through Lie algebraic arguments. We mention that the practical use of such a method might be computationally intensive, as it requires the numerical solution of a nonlinear system. In our case, however, the nilpotent system (9) to be steered is also strictly triangular. Hence, for a specific NA, a steering control satisfying the conditions of Step D2 (including Hölder-continuity in $z^{f}$ ) can be often found by exploiting the possibility of integrating in closed form the system equations under finite-time parameterized steering controls (e.g., sinusoidal, piecewise-constant, polynomial). For this reason, we shall not discuss further the computation of steering controls in the general case, referring the reader to the case study of Section V for details. 


\section{B. Control Algorithm}

Assume that a steering controller has been built following the Design Algorithm. A Control Algorithm that leads system (1) from $x_{0}$ to the origin in feedback is the following.

Step C0) Set $k=0$. The initial time and state are, respectively, $t_{0}$ and $x\left(t_{0}\right)=x_{0}$.

Step C1) Having chosen $\eta \in(0,1]$, compute $x_{k}^{f}=(1-\eta) x_{k}$ (the subgoal for the current iteration) and $z_{k}^{f}=$ $\phi_{x_{k}}\left(x_{k}^{f}\right)$ (the image of $x_{k}^{f}$ in privileged coordinates centered at the current point $x_{k}$ ).

Step C2) Let $t_{k+1}=t_{k}+T$ and $I_{k+1}=\left[t_{k}, t_{k+1}\right]$. For $t \in I_{k+1}$, apply the steering control $\alpha\left(z_{k}^{f}, t-t_{k}\right)$.

Step C3) Measure $x\left(t_{k+1}\right)$ and let $x_{k+1}=x\left(t_{k+1}\right)$.

Step C4) Set $k=k+1$ and go to Step 1 .

Some remarks are in order at this point.

- Due to the use of an NA, at the end of iteration $I_{k+1}$ there is an error between the actual state $x_{k+1}$ of system (1) and the state $\hat{x}_{k+1}=x_{k}^{f}$ of the nilpotent system, i.e., the inverse image of $\hat{z}_{k+1}=z_{k}^{f}$.

- In Step C2, the nilpotent system is simply steered closer to the origin (the destination becomes exactly the origin if $\eta=1$ ). The rationale for this apparently conservative choice is that, by limiting the required reconfiguration, one can also reduce at will the steering error between the original and the nilpotent system, so as to satisfy the conditions of the IS framework (see the proof of Theorem 2 below).

- The real-time computational load of the above Control Algorithm is very limited, and consists of the computation of $z_{k}^{f}$, the image in privileged coordinates of the current subgoal, through the change of coordinates $\phi_{x_{k}}$; the latter is simply the evaluation at $x_{k}$ of the symbolic expression $\phi_{\bar{x}}$ obtained during Step D1. Note, in particular, that the NA at $x_{k}$ is not computed. We directly use the steering controller that has been identified during the design phase.

- In Step C1, the subgoal is chosen along the line joining $x_{k}$ to the origin. In some cases, however, it may be easier to obtain error contraction along a different direction; to this end, Step C 1 can be made more general by setting $x_{k}^{f}=$ $E x_{k}$, with $E=\operatorname{diag}\left\{1-\eta_{i}\right\}, \eta_{i} \in(0,1], i=1, \ldots, n$.

\section{Stability Result}

The effectiveness of the above Control Algorithm is guaranteed by the following result, which also gives a stricter lower bound on $p$ in Step D2.

Theorem 2: The Control Algorithm makes the origin of system (1) globally asymptotically stable, with an exponential convergence rate, provided that:

1) $\eta$ is sufficiently small;

2) $p>1 /\left(w_{l}+1\right)$, where $w_{l}$ is the weight associated with the privileged coordinate $z_{l}$, and $l$ is the smallest integer such that $z_{l}(T) \neq \hat{z}_{l}(T)=z_{l}^{f}$ under the application of the steering control $\alpha\left(z^{f}, t\right)$.

Proof: The thesis will be proven by showing that the steering control designed as in Steps D1-D2, and used as in Steps C1-C2, satisfies the requirements of the IS method, that is, Condition 1 of Section III-A.
First of all, it is clear that Condition 2a) yields Condition 1a). As for Condition $1 b$ ), one must show that Hölder-continuity of order $p>0$ in $z^{f}$ implies Hölder-continuity of the same order in the initial condition, i.e., during the generic $(k+1)$ th iteration, in $x_{k}$. This is a consequence of the polynomial mapping between $z$ and $x$ expressed by (6), which results in

$$
z_{k}^{f}=\phi_{x_{k}}\left(x_{k}^{f}\right)=-\eta A x_{k}+\operatorname{pol}\left(-\eta A x_{k}\right) .
$$

Since $\operatorname{pol}(\cdot)$ only includes terms of degree $\geq 2, z_{k}^{f}$ is Höldercontinuous of order 1 in $x_{k}$ at the origin, i.e.,

$$
\left|z_{k}^{f}\right| \leq \eta \nu\left|x_{k}\right|
$$

with $\nu$ a positive number including the local Lipschitz constant of $\operatorname{pol}(\cdot)$ and $|A|$, the norm of $A$ induced by the Euclidean norm. This immediately implies that Condition $1 b$ ) is satisfied.

The final step is to show that Condition 1c) (contraction) can be guaranteed for the original system, in spite of the fact that the steering controller has been designed for the NA [Condition $2 c)]$. That is, we must prove that, for sufficiently small $\eta$, there exists a $\beta<1$ such that $\left|x_{k+1}\right| \leq \beta\left|x_{k}\right|$.

Let $e_{k+1}=x_{k+1}-\hat{x}_{k+1}$, so that we have

$$
\left|x_{k+1}\right| \leq\left|\hat{x}_{k+1}\right|+\left|e_{k+1}\right|=(1-\eta)\left|x_{k}\right|+\left|e_{k+1}\right|
$$

having used Condition 2c). Using (7), the relationship between the errors in the $x$ and $z$ coordinates is obtained as

$$
\left|e_{k+1}\right|=\left|A^{-1} e_{k+1}^{z}\right|+\left|A^{-1}\left(\operatorname{pol}^{\prime}\left(z_{k+1}\right)-\operatorname{pol}^{\prime}\left(\hat{z}_{k+1}\right)\right)\right|
$$

so that

$$
\left|e_{k+1}\right| \leq(1+\lambda)\left|A^{-1}\right|\left|e_{k+1}^{z}\right|
$$

where $\lambda$ is the local Lipschitz constant for $\operatorname{pol}^{\prime}(\cdot)$. We now invoke Lemma 1 (see the Appendix), and, in particular, the estimate (23) for the error after $T$ seconds, so as to obtain

$$
\left|e_{k+1}\right| \leq(1+\lambda) \gamma\left|A^{-1}\right|\left(u_{\max }\right)^{w_{l}+1} .
$$

By hypothesis [in particular, Condition $2 b$ )], the steering control $u(t)=\alpha\left(z_{k}^{f}, t\right)$ during the $k+1$ th iteration is a Hölder-continuous vector function of $z_{k}^{f}$, so that the same is true for $u_{\max }$, the maximum value of its components over $\left[t_{k}, t_{k+1}\right]$. Equation (10) yields then

$$
u_{\max } \leq \mu\left|z_{k}^{f}\right|^{p}
$$

Plugging this inequality into (13), and using (11), we get

$$
\left|e_{k+1}\right| \leq(1+\lambda) \gamma\left|A^{-1}\right| \mu^{w_{l}+1}\left(\nu \eta\left|x_{k}\right|\right)^{p\left(w_{l}+1\right)} .
$$

Under the hypothesis $p>1 /\left(w_{l}+1\right)$, we may write

$$
\left|e_{k+1}\right| \leq f(\eta)\left|x_{k}\right|
$$

where $f(\eta)$ is a function of order $p\left(w_{l}+1\right)>1$ at zero.

The proof is completed by noting that the above estimate for $\left|e_{k+1}\right|$, together with (12), implies

$$
\left|x_{k+1}\right| \leq(1-\eta+f(\eta))\left|x_{k}\right|=\beta\left|x_{k}\right| .
$$


Since the order of $f(\eta)$ is at least two, $\beta$ is certainly smaller than one for sufficiently small $\eta$; hence, contraction is achieved also for the original system.

Having shown that our steering controller satisfies Condition 1 , we invoke Theorem 1 to establish global asymptotic stability of the origin, with exponential convergence rate that is logarithmic in $\beta$.

The following remarks are in order with respect to the two hypotheses of Theorem 2 .

- The first hypothesis is that a sufficiently small $\eta$ (the contraction rate for the NA) is used in Step C1 of our Control Algorithm. As shown in the proof, this is required to guarantee contraction (and hence, asymptotic stability) for the original system. However, the upper bound for $\eta$ that can be derived from the last step of the proof (namely, $f(\eta)<\eta$ ) will almost certainly be overly conservative. This is due to the fact that, in order to establish an existence result valid for the whole class of systems described by (1), we had to make use of general arguments pertaining to the differential geometric structure of NAs. Often, a much more significant bound on $\eta$ can be effectively computed from the equations of the specific mechanism under consideration. As a last resort, a feasible $\eta$ may be identified through a preliminary simulation study, in which one proceeds by bisection on $\eta$ until contraction is found to be preserved for the original system.

- The second hypothesis involves $l$, i.e., the index of the first component of $z$ whose final value deviates from the desired value due to the approximation error. Clearly, the higher $l$ is, the higher $w_{l}$ is, and the easier it is to obtain $p>1 /\left(w_{l}+1\right)$. It is, therefore, convenient to perform system manipulations that can increase $l$ before designing the steering controller. In particular, if system (1) can be put $^{3}$ in the following form:

$$
\begin{aligned}
\dot{x}_{1} & =u_{1} \\
\vdots & \\
\dot{x}_{m} & =u_{m} \\
\dot{x}_{m+1} & =\sum_{j=1}^{m} g_{m+1, j}\left(x_{1}, \ldots, x_{m}\right) u_{j} \\
\dot{x}_{m+2} & =\sum_{j=1}^{m} g_{m+2, j}\left(x_{1}, \ldots, x_{m+1}\right) u_{j} \\
& \vdots \\
\dot{x}_{n} & =\sum_{j=1}^{m} g_{n, j}\left(x_{1}, \ldots, x_{n-1}\right) u_{j}
\end{aligned}
$$

then it is $l=m+1$, because the first $m$ dynamic equations (and the associated state variables $x_{j}=z_{j}, j=1, \ldots, m$ ) will be exactly represented in the NA-an obvious fact whose proof we leave to the reader. Even in the most general case, however, one can always obtain $l=2$ by feedback linearization of a single equation.

${ }^{3}$ Conditions for achieving this structure (a special case of strictly triangular form) by feedback transformations are given, e.g., in [21].

\section{Discussion}

Theorem 2 shows that the proposed stabilization technique is effective for driftless nonholonomic systems whose NA can be steered from 0 to $z^{f}$ by a control law that is Hölder-continuous of order $p>1 /\left(w_{l}+1\right)$ in $z^{f}$. After the previous discussion on what can be done to increase $w_{l}$, the reader may now wonder if there exists a structural upper bound on $p$ that must be kept in account. Although a rigorous study of this issue is outside the scope of this paper, we conjecture ${ }^{4}$ that such an upper bound is $1 / r$, where $r$ is the degree of nonholonomy of the system. As a consequence, our stabilization method provides a solution whenever $w_{l}>r-1$ can be obtained.

Altogether, the stabilizing controller resulting from our strategy belongs to the class of non-Lipschitz, Hölder-continuous time-varying feedback, and achieves asymptotic stability with exponential convergence rate; in particular, the origin is $\rho$-exponentially stable in the sense of [14]. These features are obviously in accordance with the theoretical results in the literature, and, in particular, the theorem by Brockett [4], which implies that continuous, time-invariant stabilization of driftless nonholonomic systems is impossible, and the analysis in [33], which shows how the necessity of nondifferentiable feedback extends to the time-varying case whenever exponential convergence is desired.

Concerning the robustness of the proposed stabilization technique, [19, Th. 2] indicates that the controllers based on the IS paradigm produce ultimately bounded errors under small persistent additive perturbations, while small nonpersistent perturbations are rejected, provided that they satisfy an additional condition. Due to space limitations, this issue will not be further analyzed in this paper. However, we shall illustrate the controller performance in perturbed conditions for the case study of the next section.

Before proceeding with the case study, we are now in the position to provide a more detailed comparison between our framework and the related techniques available in the literature for the stabilization of general nonholonomic systems. Apart from the methods in [36], which needs a special condition on the growth vector which would rule out, e.g., the plate-ball system, and [24], that requires a smooth stabilizing control to be known beforehand, papers [26] and [27] appear to be the closest to our approach. While the first stabilizing controller is not robust with respect to unmodeled dynamics, robustness is achieved in the second controller essentially by adopting an iteration mechanism similar to the one in this paper. In both cases, however, the steering controls are those of [17], which may be unnecessarily complicated, and cause practical problems due to their highly oscillatory nature.

In comparison, our stabilization scheme can use very simple steering controls, because they are required to move the NA of the system to a given point ( $z_{k}^{f}$ in the Control Algorithm), as

\footnotetext{
${ }^{4}$ The idea behind the proof of this result is, however, clear. If a system has degree of nonholonomy $r$, a basis for the associated Lie algebra will include at least a Lie bracket of order $r$. Hence, a steering controller for such a system must necessarily generate motion in the direction of this bracket. In particular, a well-known computation based on the method of iterated integrals [42] shows that, roughly speaking, the amount of motion obtained is a function of order $r$ in the control energy. By inverting this relationship, one should be able to show that a steering controller cannot have a Hölder-continuity order larger than $1 / r$.
} 


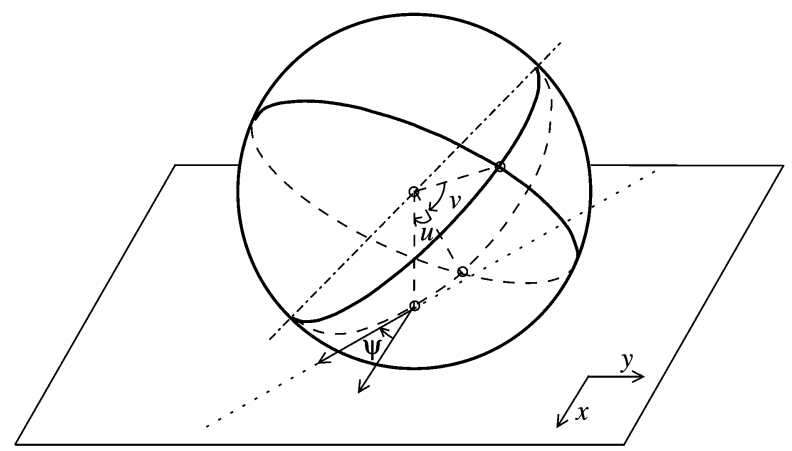

Fig. 1. Plate-ball system. The upper plate is not shown in the figure for the sake of clarity.

opposed to driving the original system along a given trajectory as in [17]. In particular, the controls do not need to be oscillatory in our case. Although in the case study, we shall use sinusoidal controls, many other choices were available, e.g., polynomial or piecewise-constant. In this sense, our strategy is more similar to the iteration of steering controls of [15], which, however, is not developed into a stabilization scheme, and of [3], where robust stabilization is achieved for the special class of chained-form systems.

Another difference between the two approaches lies in the regularity requirements imposed on the dynamics. While [27] relies on the use of a homogeneous NA of the system, hence preventing the stabilization at points where the growth vector changes, our technique can be modified for this case by using nonhomogeneous NAs [47]; see the concluding section for additional details.

\section{CAse Study: The Plate-Ball System}

In the last decade, rolling manipulation has attracted the interest of robotic researchers as a convenient way to obtain dexterity with a relatively simple mechanical design (see [22], [31], and the references therein). Controllability of the manipulation system (hand+manipulated object) with a low number of actuators is achieved thanks to the nonholonomic nature of rolling contacts between rigid bodies. This approach to manipulation can be considered as another example of the minimalistic trend in robotics, aimed at performing complex tasks through devices of reduced complexity.

The archetypal example of rolling manipulation is the plateball system, shown in Fig. 1 and consisting of a spherical ball of radius $\rho$ rolling between two horizontal plates: the lower plate is fixed, while the upper is actuated and can translate horizontally. This mechanism is controllable, i.e., the ball (the manipulated object) can be brought to any contact configuration by maneuvering the upper plate (the first finger). However, it is well known that such a system is not flat; it is, in other words, an instance of a general nonholonomic system.

To this date, we know how to steer the system between given configurations using, e.g., the numerical algorithm of [22], the symbolic algorithm of [16], or the optimal paths of [12]. As for any planner based on open-loop control, however, the successful execution of maneuvers is not preserved in the presence of perturbations; some sort of feedback is necessary to induce a degree of robustness. In [34], we attacked the stabilization problem using the IS paradigm, laying the foundations of this work. In this section, we further develop the controller therein proposed, rigorously proving that feedback stabilization of the plate-ball mechanism can be achieved through the general strategy outlined in the previous section.

Recently, feedback control of the plate-ball manipulation system was also independently addressed in [7] and [28]. In these works, however, only local convergence, not asymptotic stability in the sense of Lyapunov, is shown. From our viewpoint, it is, however, interesting that some sort of IS mechanism was used in both cases.

\section{A. Kinematic Model}

Denote by $u$ and $v$ the coordinates (latitude and longitude, respectively) of the contact point on the ball, by $x, y$ the Cartesian coordinates of the contact point on the lower plane, and by $\psi$ the angle between the $x$ axis and the plane of the meridian through the contact point (see Fig. 1). The configuration of the plate-ball system is, therefore, completely described by $q=$ $(u, v, \psi, x, y)$. There is a representation singularity when $u=$ $\pm \pi / 2$, where $v$ and $\psi$ are undefined. We assume $-\pi / 2<u<$ $\pi / 2$ and $-\pi<v<\pi$, so that the contact point belongs always to the same coordinate patch for the ball.

Under the perfect rolling assumption, the manipulation system is completely described by the kinematics of contact between the ball and the lower plate. Following the machinery due to Montana [25], one obtains

$$
\begin{aligned}
\dot{u} & =\frac{\cos \psi}{\rho} w_{x}-\frac{\sin \psi}{\rho} w_{y} \\
\dot{v} & =-\frac{\sin \psi}{\rho \cos u} w_{x}-\frac{\cos \psi}{\rho \cos u} w_{y} \\
\dot{\psi} & =\frac{\tan u \sin \psi}{\rho} w_{x}+\frac{\tan u \cos \psi}{\rho} w_{y} \\
\dot{x} & =w_{x} \\
\dot{y} & =w_{y}
\end{aligned}
$$

where $w_{x}$ and $w_{y}$ are the Cartesian components of the translational velocity of the ball, which we may assume to be directly controlled. ${ }^{5}$ Note that the above kinematic model is not valid when $u= \pm \pi / 2$.

Denote by $g_{1}$ and $g_{2}$ the input vector fields of system (14). Controllability is readily established by noting that the vector space

$$
\begin{aligned}
L^{3}(q)=\operatorname{span}\left\{g_{1}(q), g_{2}(q),\right. & {\left[g_{1}, g_{2}\right](q), } \\
& {\left.\left[g_{1},\left[g_{1}, g_{2}\right]\right](q),\left[g_{2},\left[g_{1}, g_{2}\right]\right](q)\right\} }
\end{aligned}
$$

has constant dimension five outside the representation singularity. Therefore, the plate-ball system has growth vector $(2,3$, 5) and degree of nonholonomy $r=3$.

Applying the necessary and sufficient conditions of [30], it is verified that system (14) cannot be transformed in chained form,

${ }^{5}$ Consider that the translational velocity of the ball is half the translational velocity of the upper plane. 
essentially due to the "double jump" in the growth vector. ${ }^{6}$ As this is a two-input driftless system, this also rules out flatness; alternatively, one may directly show that the necessary and sufficient conditions [37] for flatness are violated.

Our objective is to design a feedback stabilization scheme for the plate-ball system based on the IS paradigm. In principle, since exact planners are available for this mechanism, the NA step could be avoided. To build a steering controller that satisfies Condition 1 of Section III-A, one could choose a planner from [16] or [22] and modify it so as to guarantee Hölder-continuity with respect to the desired reconfiguration. We shall not pursue this approach for two reasons. First, such a modification is not trivial ${ }^{7}$ and may even prove impossible for some planners. Second, we wish to explore the potential of the stabilization strategy presented in Section IV. Therefore, an NA of the system will be derived for computing a steering controller.

To simplify the design of the stabilizing controller, it is, however, convenient to perform a preliminary feedback transformation, defined by the following change of coordinates:

$$
\begin{aligned}
& \chi_{1}=-v \\
& \chi_{2}=\sin u \\
& \chi_{3}=\psi \\
& \chi_{4}=x \\
& \chi_{5}=y
\end{aligned}
$$

and input transformation

$$
\left(\begin{array}{l}
w_{x} \\
w_{y}
\end{array}\right)=\left(\begin{array}{cc}
\sin \psi \cos u & \frac{\cos \psi}{\cos u} \\
\cos \psi \cos u & -\frac{\sin \psi}{\cos u}
\end{array}\right)\left(\begin{array}{l}
w_{1} \\
w_{2}
\end{array}\right) .
$$

Note that the above input transformation is always defined, except for $u= \pm \pi / 2$, which is, however, outside our coordinate patch.

The transformed system is strictly triangular

$$
\begin{aligned}
& \dot{\chi}_{1}=w_{1} / \rho \\
& \dot{\chi}_{2}=w_{2} / \rho \\
& \dot{\chi}_{3}=\chi_{2} w_{1} / \rho \\
& \dot{\chi}_{4}=\sin \chi_{3} \cos \left(\arcsin \chi_{2}\right) w_{1}+\frac{\cos \chi_{3}}{\cos \left(\arcsin \chi_{2}\right)} w_{2} \\
& \dot{\chi}_{5}=\cos \chi_{3} \cos \left(\arcsin \chi_{2}\right) w_{1}-\frac{\sin \chi_{3}}{\cos \left(\arcsin \chi_{2}\right)} w_{2} .
\end{aligned}
$$

In particular, note that the first three equations are in chained form.

In the following, we assume $\rho=1$ without loss of generality. This simply means that the radius of the ball defines the unitary length.

\section{B. Control Design}

Assume that we wish to transfer the plate-ball system from $q^{i}$ to $q^{d}$, respectively, the initial and desired contact configuration. To be consistent with our formulation, we assume

\footnotetext{
${ }^{6}$ System (14) represents an example of E. Cartan's famous problem of three constraints and five variables [5].

${ }^{7} \mathrm{~A}$ first step in this direction is presented in [35].
}

$q^{d}=(0,0,0,0,0) ;$ this can always be achieved by properly defining the reference frames on the ball and the lower plane. The transformed system (15) must then be transferred from $\chi^{i}=\left(\chi_{1}^{i}, \ldots, \chi_{5}^{i}\right)$ (the image of $\left.q^{i}\right)$ to the origin (the image of $\left.q^{d}\right)$

In view of the particular structure of system (15), the general stabilization framework of Section IV suggests a two-phase control strategy.

I) Drive the first three variables $\chi_{1}, \chi_{2}$, and $\chi_{3}$ to zero in finite time. Denote by $\chi^{\mathrm{I}}=\left(0,0,0, \chi_{4}^{\mathrm{I}}, \chi_{5}^{\mathrm{I}}\right)$ the system configuration at the end of this phase.

II) Apply the iterative Control Algorithm of Section IV-B to obtain exponential convergence of $\chi_{4}, \chi_{5}$ to zero while cycling over $\chi_{1}, \chi_{2}, \chi_{3}$.

Below, we describe in detail the structure of each phase.

1) Phase I Controller: To steer $\chi_{1}, \chi_{2}$, and $\chi_{3}$ from their initial values to zero (which amounts to steering the ball to the desired contact configuration $u=v=\psi=0$ regardless of $x$ and $y$, i.e., of the Cartesian position of the contact point), one can exploit the fact that the first three equations of system (15) are in chained form. Many steering controllers have been proposed for chained-form systems; below, we introduce a new controller, inspired by [44] but modified so as to guarantee the Höldercontinuity property which will be essential in the following.

For $t \in\left[0, T_{1}\right]$, let

$$
\begin{aligned}
& w_{1}=a_{0}^{\mathrm{I}}+a_{1}^{\mathrm{I}} \cos \omega t \\
& w_{2}=b_{0}^{\mathrm{I}}+b_{1}^{\mathrm{I}} \sin \omega t
\end{aligned}
$$

with $a_{0}^{\mathrm{I}}, a_{1}^{\mathrm{I}}, b_{0}^{\mathrm{I}}, b_{1}^{\mathrm{I}} \in \mathbb{R}$, and $\omega=2 \pi / T_{1}$. Choose the control parameters as follows

$$
\begin{aligned}
& a_{0}^{\mathrm{I}}=-\chi_{1}^{i} / T_{1} \\
& b_{0}^{\mathrm{I}}=-\chi_{2}^{i} / T_{1} \\
& b_{1}^{\mathrm{I}}=-2 \frac{2 \pi \chi_{3}^{i}-\pi \chi_{1}^{i} \chi_{2}^{i}+T_{1} a_{1}^{\mathrm{I}} \chi_{2}^{i}}{T_{1}^{2} a_{1}^{\mathrm{I}}} .
\end{aligned}
$$

It is easy to verify that forward integration of system (15) under this control gives $\chi_{1}\left(T_{1}\right)=\chi_{2}\left(T_{1}\right)=\chi_{3}\left(T_{1}\right)=0$, regardless of the choice of $a_{1}^{\mathrm{I}}$.

In particular, choosing

$$
a_{1}^{\mathrm{I}}=\sigma\left|\chi^{i}\right|^{1 / \delta^{\mathrm{I}}} \quad \sigma>0 \quad \delta^{\mathrm{I}}>1
$$

we obtain a steering controller that is Hölder-continuous of order $\min \left(1 / \delta^{\mathrm{I}}, 1-1 / \delta^{\mathrm{I}}\right)$. The maximum degree of continuity $1 / 2$ is attained by letting $\delta^{\mathrm{I}}=2$.

2) Phase II Controller: The iterative feedback controller of Phase II is obtained by following the general framework of Section IV. Hence, we first apply the Design Algorithm of Section IV-A to identify a steering controller, and then we provide an explicit algorithm for Phase II following the Control Algorithm of Section IV-B.

Nilpotent Approximation: Considering that Phase II starts at $\chi^{\mathrm{I}}=\left(0,0,0, \chi_{4}^{\mathrm{I}}, \chi_{5}^{\mathrm{I}}\right)$, and cycles over the first three coordinates, it is sufficient to compute the NA of the system at points of the form $\left(0,0,0, \bar{\chi}_{4}, \bar{\chi}_{5}\right)$. Following the procedure of [2], privileged coordinates for system (15) are obtained by a linear 
change of variables [compare with the general polynomial expression (6)]

$$
\begin{aligned}
& z_{1}=\chi_{1} \\
& z_{2}=\chi_{2} \\
& z_{3}=\chi_{3} \\
& z_{4}=\chi_{2}-\left(\chi_{4}-\bar{\chi}_{4}\right) \\
& z_{5}=-\chi_{1}+\left(\chi_{5}-\bar{\chi}_{5}\right) .
\end{aligned}
$$

This transformation is globally valid, a consequence of the fact that the degree of nonholonomy is three everywhere.

The NA, whose state vector is denoted by $\hat{z}$, is then computed by following the algorithm of Section III-B

$$
\begin{aligned}
& \dot{\hat{z}}_{1}=w_{1} \\
& \dot{\hat{z}}_{2}=w_{2} \\
& \dot{\hat{z}}_{3}=\hat{z}_{2} w_{1} \\
& \dot{\hat{z}}_{4}=-\hat{z}_{3} w_{1}-\frac{1}{2} \hat{z}_{2}^{2} w_{2} \\
& \dot{\hat{z}}_{5}=-\frac{1}{2} \hat{z}_{2}^{2} w_{1}-\hat{z}_{3} w_{2} .
\end{aligned}
$$

As entailed by the general form (9), the approximation is indeed polynomial and strictly triangular; note that the dynamics of $z_{1}, z_{2}, z_{3}$ coincides with that of $\hat{z}_{1}, \hat{z}_{2}, \hat{z}_{3}$. Hence, the index of the first component of $z$ affected by an approximation error is $l=4$. The corresponding weight, computed as in Definition 4 of Section III-B, is $w_{l}=3$.

Another NA for the plate-ball system is given in [1].

Steering Control: We now design an open-loop control to transfer in a finite time $T_{2}$ the NA (20) from $z=0$ to $z^{f}=$ $\left(0,0,0, z_{4}^{f}, z_{5}^{f}\right)$ (we may take this particular form for $z^{f}$ in view of the cyclicity of Phase II). To guarantee the applicability of the iterative Control Algorithm of Section IV-B, such open-loop control must satisfy Condition 2 of Section IV-A.

Define the control inputs as

$$
\begin{aligned}
& w_{1}=a_{1}^{\mathrm{II}} \cos \omega t+a_{2}^{\mathrm{II}} \cos 4 \omega t \\
& w_{2}=b_{1}^{\mathrm{II}} \cos 2 \omega t
\end{aligned}
$$

with $a_{1}^{\mathrm{II}}, a_{2}^{\mathrm{II}}, b_{1}^{\mathrm{II}} \in \mathbb{R}$ and $\omega=2 \pi / T_{2}$. Choosing the parameters as

$$
\begin{aligned}
& a_{1}^{\mathrm{II}}=\left(\frac{z_{4}^{f}}{k_{1} b_{1}^{\mathrm{II}}}\right)^{1 / 2} \\
& a_{2}^{\mathrm{II}}=\frac{z_{5}^{f}}{k_{2}\left(b_{1}^{\mathrm{II}}\right)^{2}}
\end{aligned}
$$

where $k_{1}=T_{2}^{3} / 32 \pi^{2}$ and $k_{2}=-T_{2}^{3} / 128 \pi^{2}$, forward integration of system (20) gives $\hat{z}\left(T_{2}\right)=z^{f}$ (i.e., the desired steering) for any value of $b_{1}^{\mathrm{II}}$, provided that $a_{1}^{\mathrm{II}}$ is a real number. Similar to Section V-B.1, we exploit this degree of freedom by letting

$$
b_{1}^{\mathrm{II}}=-\operatorname{sign}\left(z_{4}^{f}\right) \cdot\left|\left(\begin{array}{c}
z_{4}^{f} \\
z_{5}^{f}
\end{array}\right)\right|^{1 / \delta^{\mathrm{II}}} \quad \delta^{\mathrm{II}}>2
$$

thus making the steering controller Hölder-continuous of order $\min \left(1 / \delta^{\mathrm{II}}, 1-2 / \delta^{\mathrm{II}},\left(\delta^{\mathrm{II}}-1\right) / 2 \delta^{\mathrm{II}}\right)$. The maximum degree of continuity $1 / 3$ is attained by letting $\delta^{\mathrm{II}}=3$.
Iterative Controller: Phase II is realized by iterative application of the steering law (21)-(22), as prescribed by the following Control Algorithm.

Step II-0) Set $k=0$. The initial time and state are, respectively, $t_{0}=T_{1}$ and $\chi_{0}=\chi^{\mathrm{I}}=\left(0,0,0, \chi_{4}^{\mathrm{I}}, \chi_{5}^{\mathrm{I}}\right)$.

Step II-1) Chosen $\eta \in(0,1]$, compute $\chi_{k}^{f}=(1-\eta) \chi_{k}$ and $z_{k}^{f}=\phi_{\chi_{k}}\left(\chi_{k}^{f}\right)$ [the image of $\chi_{k}^{f}$ in privileged coordinates at $\chi_{k}$ computed via (19)].

Step II-2) Set $t_{k+1}=t_{k}+T$ and $I_{k+1}=\left[t_{k}, t_{k+1}\right]$. Compute the parameters $a_{1}^{\mathrm{II}}, a_{2}^{\mathrm{II}}, b_{1}^{\mathrm{II}}$ with $z_{k}^{f}$ in place of $z^{f}$ and $\delta^{\mathrm{II}}=3$, and apply the steering control (21)-(22) for $t \in I_{k+1}$.

Step II-3) Measure $\chi\left(t_{k+1}\right)$ and let $\chi_{k+1}=\chi\left(t_{k+1}\right)$.

Step II-4) Set $k=k+1$ and go to Step II-1.

\section{Correctness}

The correctness of the stabilization strategy so far presented can be formally proven.

Theorem 3: The above Phase I-Phase II Control Algorithm makes the origin of the plate-ball system (15) globally asymptotically stable, with exponential convergence rate, provided that $\eta$ is sufficiently small.

Proof: First, we invoke Theorem 2 to characterize the behavior of the plate-ball system during Phase II. Since the steering control (21)-(22) has Hölder-continuity order $p=1 / 3$, while the weight of the first approximated component of $z$ is $w_{l}=3$, the second hypothesis of the theorem is satisfied. Hence, for sufficiently small $\eta$, the system in Phase II exponentially converges from $\chi^{\mathrm{I}}$ to the origin. Moreover, Lyapunov stability guarantees that the state evolution can be arbitrarily bounded by appropriately bounding the initial condition of this phase, i.e., $\chi^{\mathrm{I}}$. In turn, $\chi^{\mathrm{I}}$ may be arbitrarily bounded by taking $\chi^{i}$ sufficiently close to the origin; this is a consequence of the Hölder-continuity of the steering control (16)-(17) and of the smoothness of system (15) outside the singularity $u= \pm \pi / 2$ [which can always be avoided by a proper choice of $\sigma$ in (18)].

Wrapping up, the plate-ball state $\chi$ exponentially converges from $\chi^{\mathrm{I}}$ to 0 , and can be bounded at all times by appropriately bounding the initial condition $\chi^{i}$; the thesis is then proven.

\section{Simulation Results}

Two simulations are now presented to show the effectiveness of the proposed stabilization strategy. In the first, perfect knowledge of the system is assumed (nominal case), while in the second, we have included a perturbation on the ball radius $\rho$ (perturbed case).

Nominal Case: In the first simulation, the ball radius $\rho=1 \mathrm{~m}$ is exactly known. The initial and desired configurations are given as $q^{i}=(\pi / 4, \pi / 2, \pi / 12,1,-0.5)$ and $q^{d}=(0,0,0,0,0)$, respectively ( $\left.\mathrm{rad}, \mathrm{rad}, \mathrm{rad}, \mathrm{m}, \mathrm{m}\right)$. Stabilization is obtained by applying the Control Algorithm of Section V-B. The control parameters are chosen as follows: $\sigma=1$ and $\delta^{\mathrm{I}}=2$ for the Phase I controller (16)-(17), whose duration is $T_{1}=1 \mathrm{~s} ; \delta^{\mathrm{II}}=3$ for the Phase II steering control (21)-(22), each iteration of which lasts $T_{2}=1 \mathrm{~s}$. The subgoal 


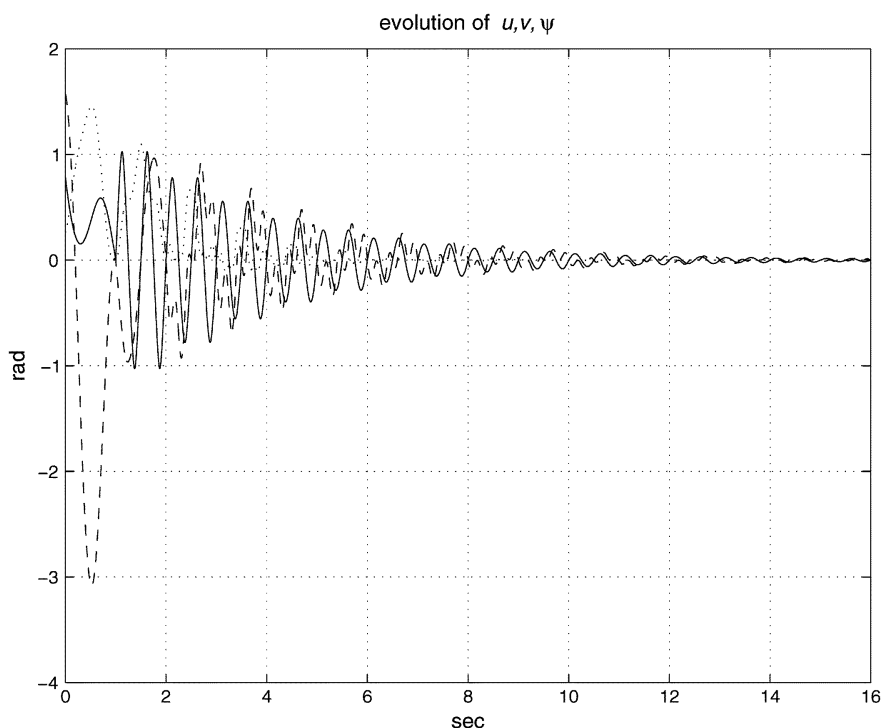

Fig. 2. Nominal case: Evolution of $u$ (solid), $v$ (dashed), and $\psi$ (dotted).

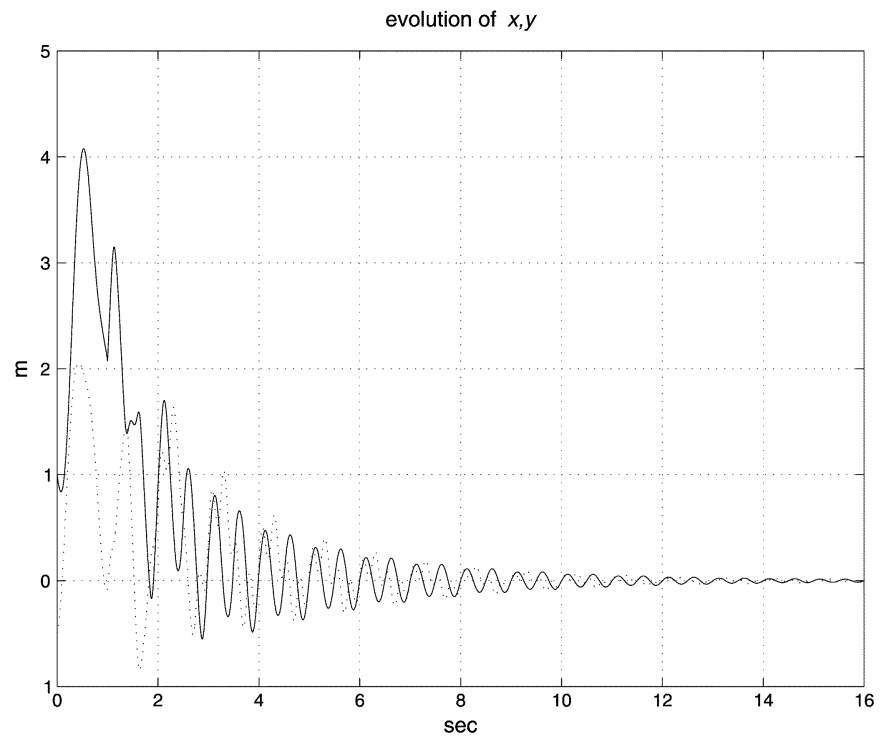

Fig. 3. Nominal case: Evolution of $x$ (solid) and $y$ (dotted).

for each iteration is computed according to Step II-1 with $\eta=0.6$.

Figs. 2 and 3 illustrate the exponential convergence of the state variables along the iterations; in particular, $u, v$, and $\psi$ are brought to zero during Phase I, while along the iterations of Phase II, they cycle around the origin on progressively contracting trajectories. The complete Cartesian path of the contact point on the plane is shown in Fig. 4; note how the paths during the iterations of Phase II become increasingly alike, although "shrinking" with time. The contraction of the positioning error is more visible in Fig. 5, which reports the path of the contact point during iterations $2,5,8$, and 11 .

Perturbed Case: In the presence of a perturbation on the ball radius, the Control Algorithm of Section V-B is not robust, essentially because Phase I is performed with the open-loop control (16)-(17), which is exact only for the nominal value of $\rho$, i.e., 1. However, it is possible to modify the Control Algorithm

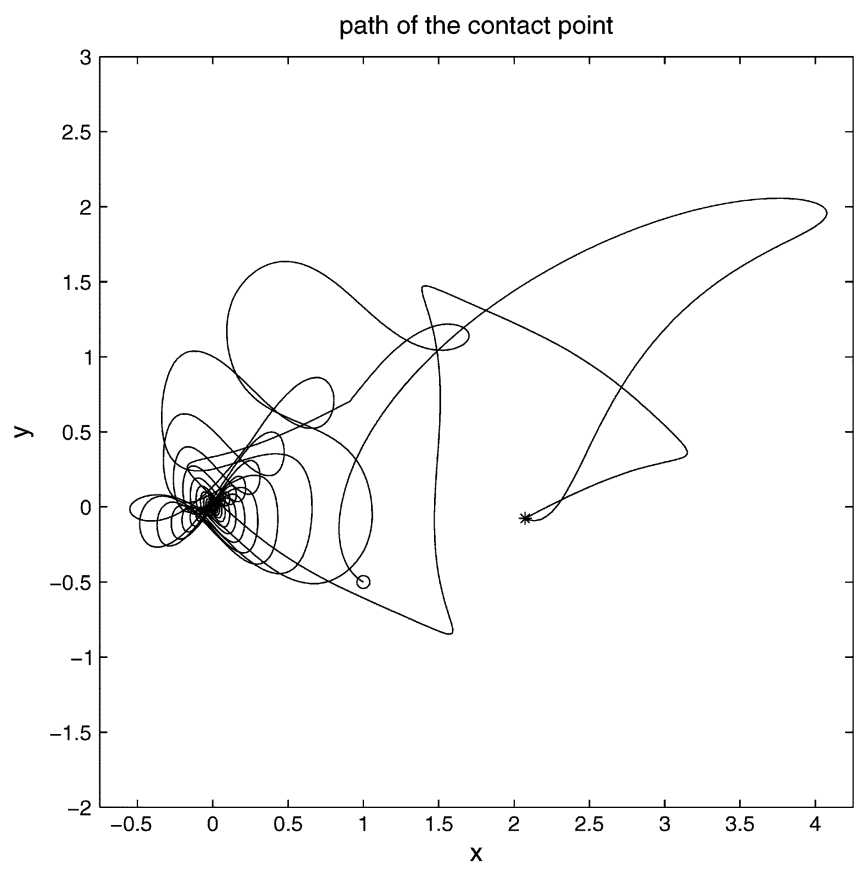

Fig. 4. Nominal case: Cartesian path of the contact point (the small circle indicates $q^{i}$, the asterisk indicates $q^{\mathrm{I}}$ ).
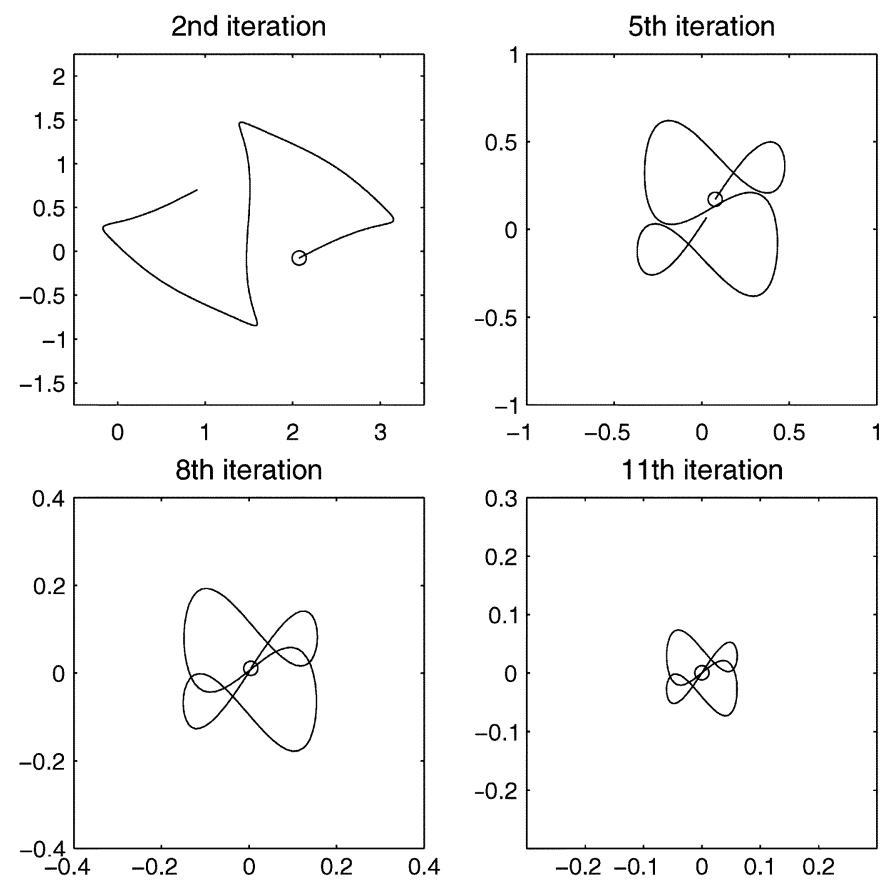

Fig. 5. Nominal case: Cartesian paths of the contact point during the second, fifth, eighth, and eleventh iterations (the small circle indicates the starting point of each iteration). Notice the different scale in the plots.

in such a way that robustness is achieved; it is sufficient to iterate Phase I, as well. Therefore, a single iteration prescribes the application of a two-phase steering control, whose expression is given by (16)-(17) during the first $T_{1}$ seconds, and by (21)-(22) during the last $T_{2}$ seconds. Note that the modified algorithm reduces to the original stabilizer of Section V-B when no perturbation is present. Robustness can be established on the basis of the nominal system stability, since the requisites of [19, Th. 2] for disturbance rejection are met. In particular, it is easy to verify 


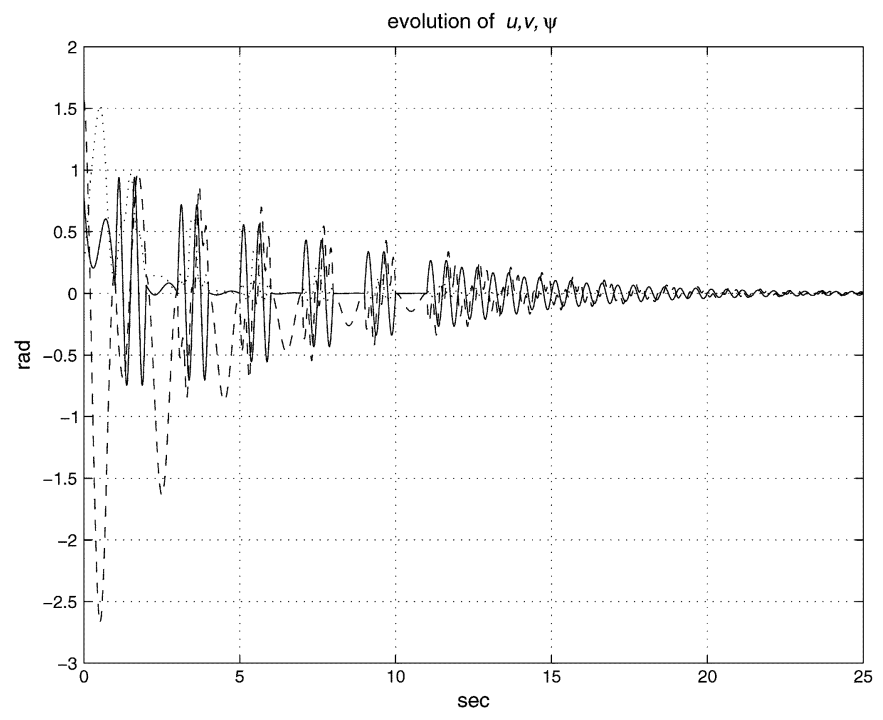

Fig. 6. Perturbed case: Evolution of $u$ (solid), $v$ (dashed), and $\psi$ (dotted).

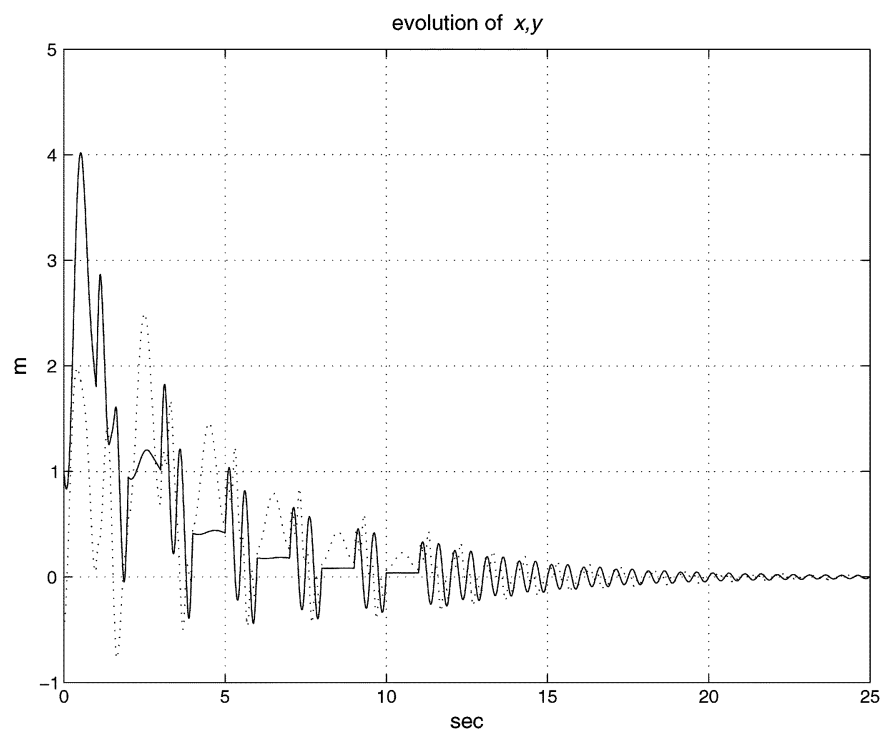

Fig. 7. Perturbed case: Evolution of $x$ (solid) and $y$ (dotted).

that the uncertainty on the ball radius can be modeled as an additive perturbation.

The second simulation confirms the robustness achieved by the modified algorithm. Here, $q^{i}, q^{d}$, as well as the control parameters, are the same as the previous simulation, but a $10 \%$ perturbation on the value of the ball radius has been introduced; the nominal unit value is used for computing the control laws, while in simulation, we have set $\rho=1.1 \mathrm{~m}$. Figs. 6 and 7 confirm that exponential convergence is preserved despite the perturbation. Note that each iteration is now composed of two phases and lasts, in principle, $T_{1}+T_{2}=2 \mathrm{~s}$. However, in practice, the first phase is not executed when the error on $u, v, \psi$ is below a given tolerance; in the present simulation, this happens starting with the seventh iteration (i.e., from $t=12^{+}$). The complete Cartesian path of the contact point on the plane is shown in Fig. 8, while Fig. 9 shows the paths during iterations 2, 5, 8, and 11. Consider that iterations 2 and 5 contain now a repetition of Phase I, as well.

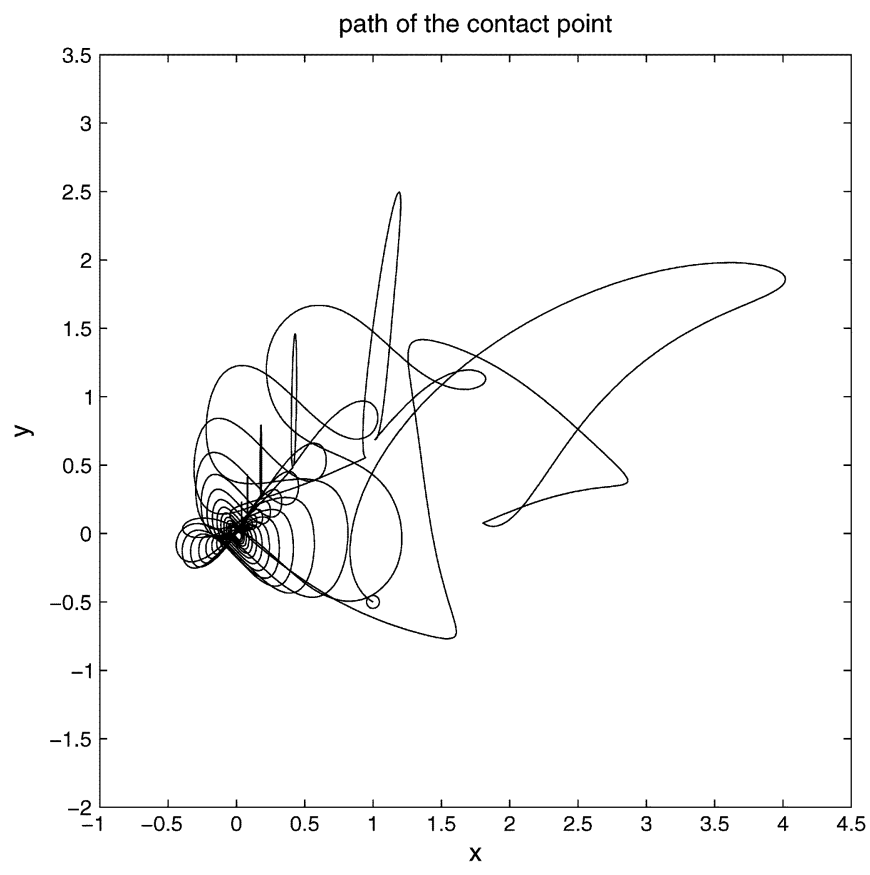

Fig. 8. Perturbed case: Cartesian path of the contact point (the small circle indicates $q^{i}$ ).
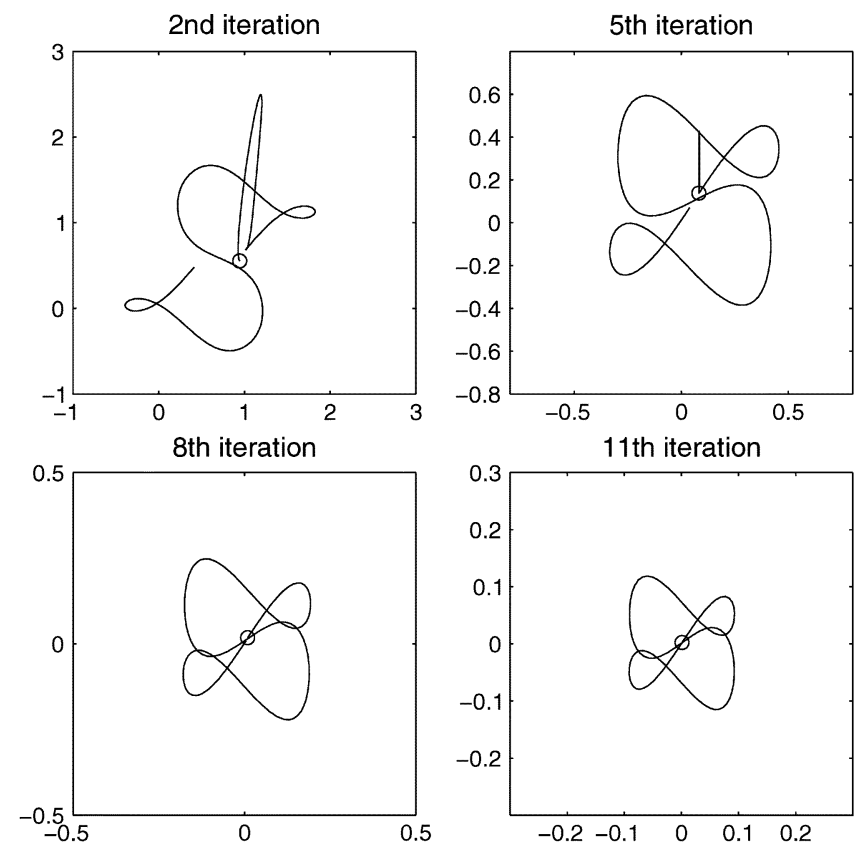

Fig. 9. Perturbed case: Cartesian paths of the contact point during the second, fifth, eighth, and eleventh iterations (the small circle indicates the starting point of each iteration). Notice the different scale in the plots.

\section{CONCLUSION}

We have presented a framework for stabilization of general nonholonomic systems, i.e., driftless controllable systems that do not possess special properties, such as exact nilpotentizability or flatness. Our strategy makes use of two tools: an iterative control scheme and a nilpotent approximation of the system dynamics. The latter is used to compute an approximate steering control which, applied repeatedly to the system, 
guarantees asymptotic stability with exponential convergence to any desired set point under the conditions of Theorem 2 .

As a case study, we have applied the proposed strategy to design a stabilizing controller for the plate-ball manipulation system, a canonical example of general nonholonomic mechanism. The theoretical performance (including exponential convergence) of the algorithm has been confirmed by simulations, both in the nominal case and in the presence of a perturbation on the ball radius. Another application of the same general framework can be found in [46], where we have designed a stabilizing controller for a system composed by a car towing two off-hooked trailers.

We conclude the paper by mentioning some issues that will be the subject of future work.

- The proposed strategy is based on the design of a suitable approximate steering control. Essentially, Hölder-continuity of an appropriate order is required w.r.t. the desired reconfiguration (see Theorem 2). Such order is related to the differential-geometric structure of the system, and, in particular, to the weight of the first approximated component in privileged coordinates. A characterization of the maximum order of continuity attainable for a system having a certain degree of nonholonomy would be useful; a starting point is sketched in Footnote 4.

- An additional difficulty arises when the considered nonholonomic system has singular points, i.e., points around which the system growth vector is not constant. For example, this is the case of the car towing two off-hooked trailers, which is singular when the trailers align with the car. Around singularities, the vector fields of the NA do not vary continuously with the approximation point. As a consequence, different steering controls must be used, and the estimate of the approximation error is not uniform, making the proposed stabilization scheme inherently local. One way to circumvent this problem is to make use of nonhomogeneous NAs [47], which possess the required continuity and uniformity properties.

- Since we have considered only driftless dynamics, the method proposed in this paper can be successfully applied to nonholonomic mechanisms whose control can be effectively designed on the basis of the kinematic model; a perfectly reasonable assumption in many cases, e.g., wheeled mobile robots and manipulation systems based on rolling contact. The extension to systems with drift, which would allow the application to underactuated robots (i.e., mechanisms with passive degrees of freedom), appears to be possible given that: 1) the IS paradigm does not require the system to be driftless [19]; and 2) NAs can be computed for systems with drift. For example, in [18], we have used the same approach to stabilize a $2 \mathrm{R}$ planar robot with a second passive joint at a given configuration. Clearly, much more study is needed in order to devise a method of general validity; to this end, it will also be advisable to explore the relationship between ideas used here and other concepts, such as the forced recurrence of [20].
- An advantage of the proposed technique, which can be useful for performing manipulation in the presence of obstacles, is the possibility of shaping the system trajectory during the generic iteration through the choice of the steering control.

- Apart from their use for stabilization, the steering controls designed in this paper can also be of interest when nonholonomic motion planning among obstacles is performed, following the two-phase approach of [39]. This technique requires first planning a collision-free path using a holonomic planner, and then interpolating points along the path by means of a "local planner"-i.e., a steering control. Completeness is guaranteed, provided that the local planner has the so-called topological property, which is a geometric counterpart of the Hölder-continuity property considered in this paper. Another similar concept is the local-local property of planners, defined by [23].

\section{APPENDIX}

We present here a technical lemma that gives an estimate of the error (in privileged coordinates) between the original system and its NA. This estimate is used in the proof of Theorem 2.

Lemma 1: Assume that the control system (1) is steered by a bounded finite-time control law $u(t), t \in[0, T]$, and let

$$
u_{\max }=\max _{\substack{i=1, \ldots, m \\ t \in[0, T]}}\left|u_{i}(t)\right|
$$

Let $z(T)$ and $\hat{z}(T)$ be the state of the original and nilpotent system, respectively, in privileged coordinates centered at $x(0)$. Then, there exists a positive constant $\gamma$ such that the following estimate holds:

$$
\left|e^{z}(T)\right|=|z(T)-\hat{z}(T)| \leq \gamma\left(u_{\max }\right)^{w_{l}+1}
$$

where $w_{l}$ is the weight associated with the coordinate $z_{l}$, and $l$ is the smallest integer such that $z_{l}(T) \neq \hat{z}_{l}(T)=z_{l}^{f}$ under the application of the steering control $u(t)$.

Proof: We give the essential arguments of this proof, which closely follows, for a large part, the proof of [2, Prop. 7.29].

The expression (8) of the original system in privileged coordinates can be expanded as

$$
\dot{z}_{j}=\sum_{i=1}^{m} \hat{g}_{i j}\left(z_{1}, \ldots, z_{j-1}\right) u_{i}+\sum_{i=1}^{m} r_{i j}(z) u_{i}, \quad j=1, \ldots, n
$$

where (see the end of Section III-B) the $\hat{g}_{i j}$ 's are homogeneous polynomials of weighted degree $w_{j}-1$, and the remainder terms $r_{i j}(z)$ are functions of order $w_{j}$ at 0 . This gives the following differential inequality:

$$
\left|\dot{z}_{j}\right| \leq u_{\max } c_{j}\|z\|^{w_{j}-1}, \quad j=1, \ldots, n
$$


where $c_{j}$ is a suitable constant, and $\|z\|$ denotes the pseudonorm of $z$, defined as

$$
\|z\|=\sum_{j=1}^{n}\left|z_{j}\right|^{1 / w_{j}} .
$$

The pseudonorm has the property of being homogeneous of degree one w.r.t. the one-parameter group of dilations associated to $w_{1}, \ldots, w_{n}$ [2].

To integrate inequality (24), it is convenient to introduce the equivalent pseudonorm

$$
|\|z\||=\left(\sum_{j=1}^{n} z_{j}^{N / w_{j}}\right)^{1 / N}
$$

where the integer $N$ has been chosen in such a way that $N / w_{j}$ is an even integer $\forall j$. By computing the time derivative of $\||| z||$, and using the properties $\left|z_{j}\right|^{1 / w_{j}} \leq|\|z\||\|\| z,\|\leq n|\|z\||$, we obtain

$$
\|\dot{z}\| \mid \leq u_{\max } n \sum_{j=1}^{n} \frac{c_{j}}{w_{j}}=c u_{\max }
$$

with the appropriate expression of $c$. Hence, it is

$$
\|z \mid\| \leq c u_{\max } t, \quad t \in[0, T]
$$

having used the fact that $z(0)=0$. Subtracting the nilpotent dynamics (9) from the original dynamics (8), we get

$$
\begin{aligned}
\dot{z}_{j}-\dot{\hat{z}}_{j}= & \sum_{i=1}^{m} u_{i}\left(\hat{g}_{i j}\left(z_{1}, \ldots, z_{j-1}\right)\right. \\
& \left.-\hat{g}_{i j}\left(\hat{z}_{1}, \ldots, \hat{z}_{j-1}\right)+r_{i j}(z)\right) \\
= & \sum_{i=1}^{m} u_{i}\left(\sum_{k \mid w_{k}<w_{j}}\left(z_{k}-\hat{z}_{k}\right) Q_{i j k}(z, \hat{z})+r_{i j}(z)\right)
\end{aligned}
$$

where the $Q_{i j k} \mathrm{~s}$ are polynomials having weighted degree $w_{j}-$ $w_{k}-1$ w.r.t. $z$ and $\hat{z}$, or are zero.

For $j=1,(26)$ and (25) promptly give

$$
\left|\dot{z}_{1}-\dot{\hat{z}}_{1}\right| \leq u_{\max } d_{1}\|z\| \leq c n d_{1} u_{\max }^{2} t
$$

where $d_{1}$ is a suitable constant. Applying recursively the same derivation, and exploiting the strictly triangular structure of the nilpotent dynamics, one obtains an inequality for $\left|\dot{z}_{j}-\dot{\hat{z}}_{j}\right|$ which can be integrated to

$$
\left|z_{j}(T)-\hat{z}_{j}(T)\right| \leq \sigma_{j}\left(u_{\max }\right)^{w_{j}+1}, \quad j=1, \ldots, n
$$

with the appropriate expression of $\sigma_{j}$.

Since the first $l-1$ components of $z(T)$ and $\hat{z}(T)$ coincide, we have

$$
\begin{aligned}
|z(T)-\hat{z}(T)|^{2} & =\sum_{j=l}^{n}\left(z_{j}(T)-\hat{z}_{j}(T)\right)^{2} \\
& \leq \sum_{j=l}^{n}\left(\sigma_{j}\left(u_{\max }\right)^{w_{j}+1}\right)^{2} \\
& \leq\left(\gamma\left(u_{\max }\right)^{w_{l}+1}\right)^{2}
\end{aligned}
$$

with $\gamma>0$, having used (27) and the Lipschitzianity of monomial functions. The thesis immediately follows.

\section{REFERENCES}

[1] A. A. Agrachev and Y. L. Sachkov, "An intrinsic approach to the control of rolling bodies," in Proc. 38th Conf. Decision, Control, 1999, pp. 431-435.

[2] A. Bellaïche, "The tangent space in sub-Riemannian geometry," in SubRiemannian Geometry, A. Bellaïche and J.-J. Risler, Eds. Cambridge, MA: Birkhäuser, 1996, pp. 1-78.

[3] M. K. Bennani and P. Rouchon, "Robust stabilization of flat and chained systems," in Proc. 3rd Eur. Control Conf., 1995, pp. 2642-2646.

[4] R. W. Brockett, "Asymptotic stability and feedback stabilization," in Differential Geometric Control Theory, R. W. Brockett, R. S. Millman, and H. J. Sussmann, Eds. Cambridge, MA: Birkhäuser, 1983, pp. 181-191.

[5] E. Cartan, "Les systèmes de pfaff à cinq variables et les équations aux derivees partielles du second ordre," Ann. Sci. Ecole Norm., vol. 27, pp. 109-192, 1910.

[6] J.-M. Coron, "Global asymptotic stabilization of controllable systems without drift," Math. Control Signal Syst., vol. 5, pp. 295-312, 1992.

[7] H. Date, M. Sampei, M. Ishikawa, and M. Koga, "Simultaneous control of position and orientation for ball-plate manipulation problem based on time-state control form," IEEE Trans. Robot. Autom., vol. 20, pp. 465-479, Jun. 2004.

[8] J.-P. Laumond, Ed., Robot Motion Planning and Control. New York: Springer-Verlag, 1998.

[9] M. Fliess et al., "Flatness and defect of nonlinear systems: Introductory theory and examples," Int. J. Control, vol. 61, pp. 1327-1361, 1995.

[10] H. Hermes, "Nilpotent approximations of control systems and distributions," SIAM J. Control Optim., vol. 24, no. 4, pp. 731-736, 1986.

[11] — "Nilpotent and high-order approximations of vector field systems," SIAM Rev., vol. 33, pp. 238-264, 1991.

[12] V. Jurdjevic, "The geometry of the plate-ball problem," Arch. Rational Mechanics Anal., vol. 124, pp. 305-328, 1993.

[13] M. Kawski, "An angular open mapping theorem," in Analysis and Optimization of Systems, A. Bensoussan and J. L. Lions, Eds. New York: Springer, 1988, vol. 111, pp. 361-371.

[14] _ , "Homogeneous stabilizing feedback laws," Control-Theory and Advanced Technology, vol. 6, no. 4, pp. 497-516, 1990.

[15] G. Laferriere and H. J. Sussmann, "A differential geometric approach to motion planning," in Nonholonomic Motion Planning, Z. Li and J. F. Canny, Eds. Norwell, MA: Kluwer, 1992, pp. 235-270.

[16] Z. Li and J. Canny, "Motion of two rigid bodies with rolling constraint," IEEE Trans. Robot. Autom., vol. 6, pp. 62-72, Feb. 1990.

[17] W. Liu, "An approximation algorithm for nonholonomic systems," SIAM J. Control Optim., vol. 35, no. 4, pp. 1328-1365, 1997.

[18] A. De Luca, R. Mattone, and G. Oriolo, "Stabilization of an underactuated planar 2r manipulator," Int. J. Robust Nonlinear Control, vol. 10, pp. 181-198, 2000

[19] P. Lucibello and G. Oriolo, "Robust stabilization via iterative state steering with an application to chained-form systems," Automatica, vol. 37, pp. 71-79, 2001.

[20] K. M. Lynch and C. G. Black, "Recurrence, controllability, and stabilization of juggling," IEEE Trans. Robot. Autom., vol. 17, pp. 113-124, Apr. 2001.

[21] A. Marigo, "Constructive necessary and sufficient conditions for strict triangularizability of driftless nonholonomic systems," in Proc. 38th IEEE Conf. Decision, Control, 1999, pp. 2138-2143.

[22] A. Marigo and A. Bicchi, "Rolling bodies with regular surface: Controllability theory and applications," IEEE Trans. Autom. Control, vol. 45, pp. 1586-1599, Sep. 2000.

[23] _ , "A local-local planning algorithm for rolling objects," in Proc. IEEE Int. Conf. Robot. Autom., 2002, pp. 1759-1764.

[24] R. T. M'Closkey and R. M. Murray, "Exponential stabilization of driftless nonlinear control systems using homogeneous feedback," IEEE Trans. Autom. Control, vol. 42, pp. 614-628, May 1997.

[25] D. J. Montana, "The kinematics of contact and grasp," Int. J. Robot. Res., vol. 7, no. 3, pp. 17-32, 1988.

[26] P. Morin, J.-B. Pomet, and C. Samson, "Design of homogeneous timevarying feedback stabilizing control laws for driftless controllable systems via oscillatory approximation of lie brackets in closed loop," SIAM J. Control Optim., vol. 38, pp. 22-49, 1999.

[27] P. Morin and C. Samson, "Exponential stabilization of nonlinear driftless systems with robustness to unmodeled dynamics," Control, Optim., Calculus of Variations, vol. 4, pp. 1-35, 1999. 
[28] R. Mukherjee and T. Das, "Feedback stabilization of a spherical mobile robot," in Proc. IEEE Int. Conf. Intell. Robot. Syst., 2002, pp. 2154-2162.

[29] R. M. Murray, "Control of nonholonomic systems using chained forms," Fields Inst. Commun., vol. 1, pp. 219-245, 1993.

[30] — , "Nilpotent bases for a class of nonintegrable distributions with applications to trajectory generation for nonholonomic systems," Math. Controls, Signals, Syst., vol. 7, no. 1, pp. 58-75, 1994.

[31] R. M. Murray et al., A Mathematical Introduction to Robotic Manipulation. Boca Raton, FL: CRC Press, 1994.

[32] R. M. Murray and S. Sastry, "Nonholonomic motion planning: Steering using sinusoids," IEEE Trans. Autom. Control, vol. 38, pp. 700-716, Oct. 1993.

[33] R. M. Murray, G. Walsh, and S. Sastry, "Stabilization and tracking for nonholonomic systems using time-varying state feedback," in Proc. IFAC Symp. Nonlinear Control Syst. Design, 1992, pp. 182-197.

[34] G. Oriolo and M. Vendittelli, "Robust stabilization of the plate-ball manipulation system," in Proc. IEEE Int. Conf. Robot. Autom., 2001, pp. 91-96.

[35] G. Oriolo et al., "From nominal to robust planning: The plate-ball manipulation system," in Proc. IEEE Int. Conf. Robot. Autom., 2003, pp. 1759-1764.

[36] J.-B. Pomet, "Explicit design of time-varying control laws for a class of controllable systems without drift," Syst. Control Lett., vol. 18, no. 2, pp. 147-158, 1992.

[37] P. Rouchon, "Necessary condition and genericity of dynamic feedback linearization," J. Math. Syst. Estimation Control, vol. 4, no. 2, pp. 1-14, 1994.

[38] C. Samson, "Control of chained systems, application to path following and time-varying point-stabilization of mobile robots," IEEE Trans. Autom. Control, vol. 40, pp. 64-77, Jan. 1995.

[39] S. Sekhavat and J.-P. Laurnond, "Topological property for collision-free nonholonomic motion planning: The case of sinusoidal inputs for chained form systems," IEEE Trans. Robot. Autom., vol. 14, pp. 671-680, Oct. 1998.

[40] E. D. Sontag, "Control of systems without drift via generic loops," IEEE Trans. Autom. Control, vol. 40, pp. 1210-1219, Jul. 1995.

[41] O. J. Sørdalen and O. Egeland, "Exponential stabilization of nonholonomic chained systems," IEEE Trans. Autom. Control, vol. 40, pp. 35-49, Jan. 1995.

[42] H. J. Sussmann, "A general theorem on local controllability," SIAM J. Control Optim., vol. 25, no. 1, pp. 158-194, 1987.
[43] — , "New differential geometric methods in nonholonomic path finding," in Systems, Models, and Feedback: Theory and Applications, A. Isidori and T. J. Tarn, Eds. Cambridge, MA: Birkhäuser, 1992, pp. 365-384.

[44] D. M. Tilbury, "Exterior differential systems and nonholonomic motion planning," Univ. California, Berkeley, Memo. UCB/ERL M94/90, 1994.

[45] P. Vela and J. W. Burdick, "Control of underactuated drift less systems using higher-order averaging theory," in Proc. Amer. Control Conf., vol. 2, 2003, pp. 1536-1541.

[46] M. Vendittelli and G. Oriolo, "Stabilization of the general two-trailer system," in Proc. IEEE Int. Conf. Robot. Autom., 2000, pp. 1817-1822.

[47] M. Vendittelli et al., "Nonhomogeneous nilpotent approximations for systems with singularities," IEEE Trans. Autom. Control, vol. 49, pp. 261-266, Jun. 2004

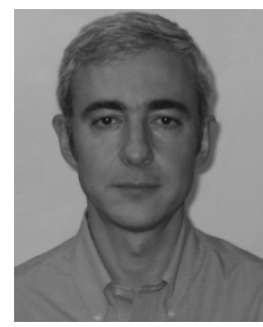

Giuseppe Oriolo (S'89-M'91-SM'02) received the "Laurea" degree in electrical engineering in 1987 and the Ph.D. degree in systems engineering in 1992, both from the University of Rome "La Sapienza," Rome, Italy.

Since 1998, he has been Associate Professor of Automatic Control, Department of Computer and System Science of the same university. His research interests are in the area of robotics and control.

Dr. Oriolo is an Associate Editor of the IEEE TRANSACTIONS ON ROBOTICS.

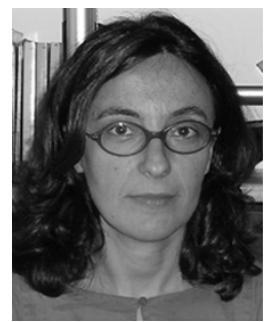

Marilena Vendittelli received the "Laurea" degree in electrical engineering in 1992 and the Ph.D. degree in systems engineering in 1997, both from the University of Rome "La Sapienza," Rome, Italy.

In November 1998, she joined the Robotics Laboratory at the Department of Computer and Systems Science (DIS), University of Rome "La Sapienza," where she currently is an Assistant Professor. Her main research interests are in the area of robot motion planning and control. 